\title{
Modeling the Interaction of a Single-Cycle Laser Pulse With a Bound Electron Without Ionization
}

\author{
Ufuk Parali and Dennis R. Alexander \\ Department of Electrical Engineering, \\ University of Nebraska Lincoln \\ Lincoln, NE 68588, \\ USA
}

\section{Introduction}

Ultrashort light pulse research has led to the creation of laser systems generating pulses only a few cycles in duration (Couairon et al., 2006). Now that these ultrashort few-cycle EM pulses exist experimentally, the need for mathematical models to describe these short pulse interactions with matter becomes very important (Porras, 1999). Questions arise on what is the meaning of the index of refraction of a material during a single cycle pulse interaction. There is a growing need to model and to understand the interaction of single ultrashort pulses or a train of ultrashort pulses with matter below the point where strong field effects dominate. This need is driven by the advances made in femtosecond (fs) and attosecond (as) laser technologies. Applications of these ultra short pulses range from free space communications, 3D depth profiling in biological samples, optical communication, high resolution/precision atomic and molecular scale imaging, high speed electronics and optoelectronics in terahertz $(\mathrm{THz})$ regime, behavior of electrons in quantum structures, relativistic physics, high-energy physics, astrophysics to medical applications. Furthermore, ultrafast few cycle lasers are expected to be a promising solution to probe the fastest events in atomic, molecular, biochemical, and solid state systems due to their unique property of being the shortest controlled bursts of energy ever developed (Corkum, 2007; Zewail, 2000; Niikura, 2002; Itatani et al., 2004; Krauss et al., 2009; Couairon et al., 2006; Yan et al., 1985; Steinmeyer et al., 1999; Akimoto, 1996).

Basic physics of the pulse-matter interaction depends strongly on the ratio of the pulse duration and the characteristic response time of the medium (as well as on the pulse intensity and energy). This ratio is the key term in the polarization response of the medium from a classical point of view. The goal of this book chapter is to provide insight in the linear polarization response of dispersive materials to ultrashort single cycle pulses. This book chapter is concerned with the case where the electric field strength is low and thus would not produce ionization. Since the energy is below the ionization threshold of the medium, there is not any plasma effect during the interaction of the applied field with the material Understanding the linear polarization response is extremely crucial in order to formulate a realistic field integral. This realistic field integral will provide a more realistic propagation model of optical pulses through dispersive media (Joseph et al. ,1991; Dvorak \& Dudley, 1995; Kozlov \& Sazabov, 1997; Wilkelmsson, et al., 1995, Kinsler, 2003; Eloy \&Wilhelmsson, 
1997; Pietrzyk et al., 2008; Macke \& Segard, 2003; Zou \& Lou, 2007; Xiao \&Oughstun 1999; Hovhannisyan, 2003. The interaction of an ultra short pulse with matter involves the interaction of the incident electric field with the electrons of the material. In this book chapter, classical approaches to this problem are modified in two separate cases for solving the interaction of a single-cycle ultrashort laser pulse with a bound electron without ionization. In this book chapter, interaction of an ultrashort single-cycle pulse (USCP) with a bound electron without ionization is compared for two different assumptions on the movement of the electron and the applied field. For a more realistic mathematical description of USCPs, Hermitian polynomials and combination of Laguerre functions are used for two different single cycle excitation cases. These single cycle pulse models are used as driving functions for the classical approach to model the interaction of a bound electron with an applied electric field. A new novel time-domain technique was developed for modifying the classical Lorentz damped oscillator model in order to make it compatible with USCP excitation (Parali \& Alexander, 2010). This modification turned the Lorentz oscillator model equation into a Hill-like function with non-periodic time varying damping and spring coefficients. In section two of this book chapter, we extend earlier work (Parali \& Alexander, 2010) by introducing a convolution of the applied electric field with the time dependent position of the electron. This latter model provides a continuous updating of the applied electric field convoluted with the time dependent position of the electrons motion. The two models vary in the complexity of the assumptions being applied to the computations. For the sake of completeness, we have chosen to include both pieces of work in this book chapter.

\subsection{Mathematical model}

In order to make an original contribution for the analysis of the interaction of an ultrashort single-cycle pulse (USCP) with a bound electron without ionization, first it is necessary to find a realistic model for a USCP. Such pulses have a rather different structure from conventional modulated quasi-monochromatic signals with a rectangular or Gaussian envelope (Shvartsburg, 1998; Wang et al., 1997; Shvartsburg, 1996; Shvartsburg, 1999). Due to the following main reasons associated with USCPs, combination of Laguerre functions and Hermitian polynomials (Mexican Hat) are used in this study for modeling applied EM field:

i. Arbitrary transient steepness: The rising and the falling times of the signal can be essentially unequal.

ii. Varying zero spacing: The distances between zero-crossing points may be essentially unequal.

iii. Both the waveform envelope and its first spatial and temporal derivatives are continuous.

iv. Arbitrary envelope asymmetry: USCP waveforms can be classified conventionally for two groups.

1. The sharply defined zero-crossing point at the pulse leading edge as initial point (combination of Laguerre functions).

2. The sharply defined narrow maximum against a background of comparatively long tails (Hermitian polynomials - Mexican Hat) (Shvartsburg, 1998; Wang et al., 1997; Shvartsburg, 1996; Shvartsburg, 1999).

Although delta function or the Heaviside step function are widely used, they assume zero signal duration and zero relaxation time. These assumptions are not suitable for modeling 
the waveform of a USCP. There are some other more realistic models, such as modulated Gaussian or rectangular transients, but these models assume equally spaced zeros which is not suitable for a USCP, neither (Shvartsburg, 1998; Wang et al., 1997; Shvartsburg, 1996; Shvartsburg, 1999).

The combination of Laguerre functions for defining the spatiotemporal profile of a USCP is defined as $E_{m}(t)=B\left(L_{m}(t)-L_{m+2}(t)\right)$ where $L_{m}(x)=(\exp (x / 2) / m !) \frac{d^{m}}{d x^{m}}\left[\exp (-x) x^{m}\right]$ is a single Laguerre function with order $\mathrm{m}$ and $x=\left(t-z c^{-1}\right) / t_{0}$. Here, $c$ is the velocity of light in vacuum, $z$ is the propagation direction and $t_{0}$ is the time scale of the pulse. In this study, the combination of $2^{\text {nd }}$ and $4^{\text {th }}$ order Laguerre functions are used to define a single USCP:

$$
E_{2}(\alpha)=\exp \left(-(7.5 \alpha)^{2}\right)\left[-\frac{1}{24} \alpha^{4}+\frac{15}{24} \alpha^{3}-\frac{5}{2} \alpha^{2}+2 \alpha\right]
$$

where the phase term is defined as $\alpha=\left(t-\phi-z c^{-1}\right) / t_{0}$ in which $\phi$ is the initial phase [Fig. $1(\mathrm{a})]$.
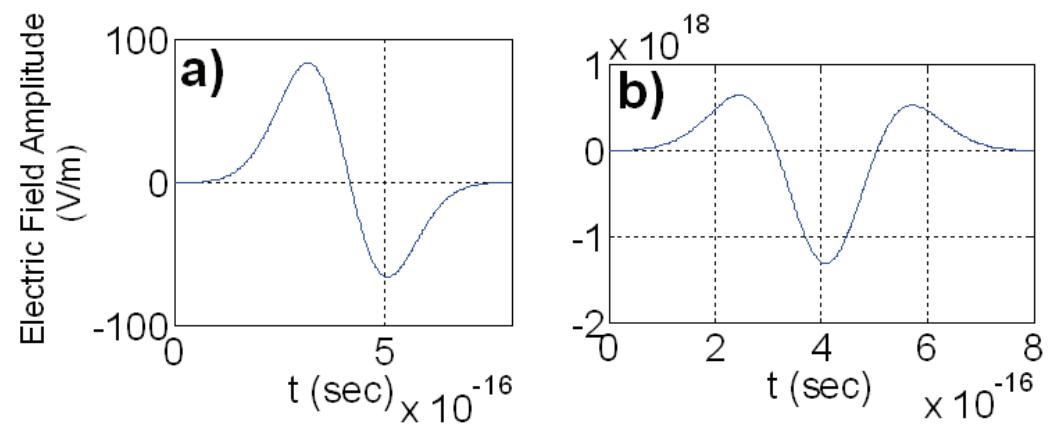

Fig. 1. (a) Applied Laguerre USCP with pulse duration $\tau_{\mathrm{p}}=8 \times 10^{-16}$. (b) $1^{\text {st }}$ derivative of the LaguerreUSCP.

Fig. 1(b) shows the first derivative of the applied field and it is clearly seen that the analytical expression $E(\alpha)$ in Eq. 1 satisfies the conditions of arbitrary transient steepness and arbitrary envelope asymmetry. From Fig. 1(a), it is also clearly seen that it satisfies the condition of varying zero spacing for a USCP. In addition to these, time profile of the Laguerre USCP is almost fulfilling the integral property:

$$
\int_{0}^{\infty} E_{2}(\alpha) d \alpha=0
$$

For the Hermitian (Mexican Hat) USCP [Fig. 2(a)], the following definition is used:

$$
E(\alpha)=\left(1-\alpha^{2}\right) \exp \left(-\alpha^{2} / 2\right)
$$

Fig. 2(b) illustrates that the Hermitian pulse satisfies the above concerns. 

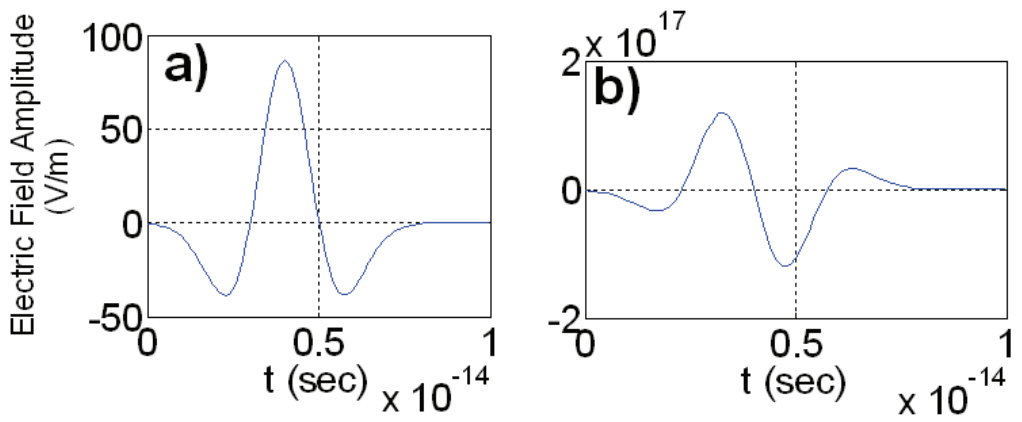

Fig. 2. (a) Applied Hermitian USCP with pulse duration $\tau_{\mathrm{p}}=8 \times 10^{-15}$, (b) $1^{\text {st }}$ derivative of the Hermitian USCP.

In addition to the question how to formulate ultrashort single cycle transients, it is also natural to ask how these pulses propagate in optical medium. In this study, USCP means the smallest possible single cycle piece (unity source) of a wave packet. It is the part of an actual carrier field and does not contain any other carrier fields in itself. For a USCP, it is difficult to introduce the concept of an envelope and it is not possible to define a group velocity. For such short pulses the distinction between carrier oscillations and slowly varying envelope (SVE), which have two different temporal scales that are peculiar to quasi-monochromatic pulses, becomes diffuse or meaningless (Xiao \& Oughstrun, 1999; Rothenberg, 1992; Humagai et al., 2003; Crisp, 1970). Jumping from many cycle optical waves to single cycle optical pulses in dealing with light-matter interaction, the mathematical treatments should be revised. The traditional analysis of pulsed EM phenomena is questionable (Shvartsburg, 1998; Wang et al., 1997; Shvartsburg, 1996; Shvartsburg, 1999). If the applied field is a USCP, the shortest possible field as explained above, then it is impossible to separate the applied source into pieces to find the effect of each part (or piece) by superposing as being suggested in the models explained in many fundamental textbooks (Scaife, 1989).

In order to understand the USCP-medium interaction phenomenon, we must acquire certain special features such as operating directly with Maxwell equations beyond the scope of Fourier representations [(Shvartsburg, 1998; Wang et al., 1997; Shvartsburg, 1996; Shvartsburg, 1999). Since the situations occur where the time scale of the pulse is equal or shorter than the relaxation time of the medium, material has no time to establish its response parameters during the essential part of the pulse continuance (Gutman, 1998; Gutman 1999; Daniel 1967; Shvartsburg 2005; Shvartsburg 2002). These parameters, which govern the polarization response of the media, change their values during the pulse continuance (Gutman, 1998; Gutman, 1999). Thus, solutions of Maxwell equations with time-dependent coefficients are required for the analysis of the wave dynamics (Shvartsburg, 2005; Shvartsburg, 2002).

In our study, we consider an approach such that under a single USCP excitation, the change in the relative position of a bound electron to its parent atom without ionization will change the amplitude of the dipole in the atom and so forth the instantaneous polarization. As a result of this fluctuation in the polarization, the index of refraction will change in the duration of the single USCP excitation during which the propagation dynamics of the same applied USCP and the other USCPs coming after the first one will be evaluated. So physically, we consider a case where the medium is including the source. This is a common situation especially in optical communication. In addition to this, we can associate this 
approach to some diagnostic techniques in ultrafast optics such as pump-probe experiments where both pump and probe pulses propagate and evaluate the time varying physical parameters of the medium. But before diving into Maxwell equations, we have to figure out how the polarization response of the medium must be handled for the interaction of a USCP EM field with a bound electron. Understanding the polarization response of the material under the excitation of a USCP EM field is one of the most important, not clearly answered yet, core question of today and near future ultrafast laser engineering.

Polarization is a very crucial physical term, especially for optical communication, since it defines the change in the index of refraction in the material due to the applied field (Gutman, 1998; Gutman, 1999; Daniel, 1967; Cole \& Cole, 1941; Djurisic \& Li, 1998). In terms of permittivity, we can write index of refraction (for a nonmagnetic material) as:

$$
n=\left(1+P_{p o l}(t) / \varepsilon_{0} E(t)\right)^{1 / 2},
$$

where $\varepsilon_{o}$ is the permittivity of free space, $E(t)$ is the applied electric field, and $P_{p o l}(t)$ is the electronic polarization. The polarization response of the medium gives the change in the index of refraction. This change or this polarization response affects the temporal and spatial evaluation of the propagating pulse (Couairon et al., 2006; Steinmeyer et al., 1999; Blanc et al., 1993; Agrawal \& Olsson 1998; Schaffer 2001).

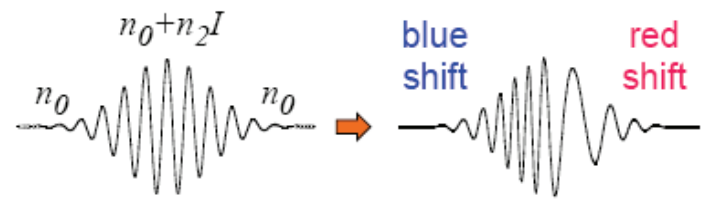

Fig. 3. Schematic representation of self modulation (pulse chirping). Although we are interested in the low intensity applied fields for linear polarization in this study, temporal dependence of the intensity profile of the applied field can still cause a temporal dependence in the refractive index (Schaffer, 2001).

The starting point of all these dynamics is the inhomogeneous wave equation:

$$
\frac{\partial^{2} E(z, t)}{\partial z^{2}}-\frac{1}{c_{0}^{2}} \frac{\partial^{2} E(z, t)}{\partial t^{2}}=\mu_{0} \frac{\partial^{2} P_{p o l}}{\partial t^{2}},
$$

where the polarization is the source term of the governing differential equation. In order to find the polarization, we must find the oscillation field (displacement) of the bound electrons. According to the Lorentz damped forced oscillator model:

$$
m_{e} \frac{d^{2} x(t)}{d t^{2}}+m_{e} \gamma_{o} \frac{d x(t)}{d t}+k_{o} x(t)=e E(t)
$$

$x(t)$ is the time dependent displacement or the oscillation field of a bound electron with respect to the applied field $E(t), \gamma_{o}$ is the damping constant, $k_{o}$ is the spring constant of the material and $m_{e}$ is the mass of electron. 
For USCP excitation, unlike the long pulse excitation fields, the response (oscillation) of the electron must be handled in a different manner. Since, both due to the mass of inertia of the electron and the shortness of the USCP compared to the relaxation time of the medium, the electron will not sense the applied field exactly at the leading edge point of the pulse. The response of the electron to the applied field will increase gradually. During this time period, the electron will not follow the oscillation profile of the applied electric field. So, the oscillation field of the electron will not only have a difference in the phase but also will have a different time profile (time-dependency) with the applied field. In regular cases, if the applied field is in the form of $e^{j w t}$ time-dependency, then we assume that the oscillation of the electron will be in the same time-dependency form. In the literature, Lorentz oscillator model is directly used in $e^{j w t}$ time-dependency (Oughstun \& Sherman 1989). But for a USCP excitation, not only the time-dependency $e^{j w t}$ is not valid, but also the oscillation field will have a different waveform than the applied field waveform (time-dependency). This means that, the $x(t)$ term in Eq. (6), that is the oscillation motion field of the electron, will have a modified form of time-dependency with respect to the applied USCP. In order to define the modified function $x(t)$, we developed a new time domain technique that we call "Modifier Function Approach". In this approach, we define the oscillation field of the electron as the multiplication of the applied USCP with the modifier function:

$$
x(t)=x_{o}(t) E(t)
$$

where $x_{o}(t)$ is the modifier function. It has a unit of (meter $)^{2} /$ volt which is equivalent to Coulomb*meter/Newton. So physically, modifier function defines dipole moment per unit force. Plugging Eq. (7) into Eq. (6), we obtain

$$
m_{e} \frac{d^{2}\left(x_{o}(t) E(t)\right)}{d t^{2}}+m_{e} \gamma_{o} \frac{d\left(x_{o}(t) E(t)\right)}{d t}+k_{o}\left(x_{o}(t) E(t)\right)=e E(t) .
$$

Performing the necessary calculations in Eq. (8), we obtain Eq. (9) and(10):

$$
\begin{gathered}
m_{e} \frac{d^{2} x_{o}(t)}{d t^{2}} E(t)+2 m_{e} \frac{d x_{o}(t)}{d t} \frac{d E(t)}{d t}+m_{e} x_{o} \frac{d^{2} E(t)}{d t^{2}}+m_{e} \gamma_{o} \frac{d x_{o}(t)}{d t} E(t)+ \\
m_{e} \gamma_{o} x_{o}(t) \frac{d E(t)}{d t}+k_{o} x_{o}(t) E(t)=e E(t) \\
m_{e} E(t) \frac{d^{2} x_{o}(t)}{d t^{2}}+m_{e}\left(2 \frac{d E(t)}{d t}+\gamma_{o} E(t)\right) \frac{d x_{o}(t)}{d t}+m_{e}\left(\frac{d^{2} E(t)}{d t^{2}}+\gamma_{o} \frac{d E(t)}{d t}+\frac{k_{o}}{m_{e}} E(t)\right) x_{o}(t)=e E(t) \\
\frac{d^{2} x_{o}(t)}{d t^{2}}+\left(\frac{2}{E(t)} \frac{d E(t)}{d t}+\gamma_{o}\right) \frac{d x_{o}(t)}{d t}+\left(\frac{1}{E(t)} \frac{d^{2} E(t)}{d t^{2}}+\frac{\gamma_{o}}{E(t)} \frac{d E(t)}{d t}+\frac{k_{o}}{m_{e}}\right) x_{o}(t)=\frac{e}{m_{e}}
\end{gathered}
$$

We can briefly write Eq. (10) as:

$$
\frac{d^{2} x_{o}(t)}{d t^{2}}+P(t) \frac{d x_{o}(t)}{d t}+Q(t) x_{o}(t)=\frac{e}{m_{e}}
$$


where

$$
\begin{gathered}
P(t)=\frac{2}{E(t)} \frac{d E(t)}{d t}+\gamma_{o}, \\
Q(t)=\frac{1}{E(t)} \frac{d^{2} E(t)}{d t^{2}}+\frac{\gamma_{o}}{E(t)} \frac{d E(t)}{d t}+\frac{k_{o}}{m_{e}} .
\end{gathered}
$$

It is seen from Eq. (11) that this equation has a similar form to that of a Hill type equation where for a regular Hill equation, $P(t)$ and $Q(t)$ terms are periodic and the right side is zero. A linear equation of this type occurs often when a system exhibiting periodic motion is perturbed in some way (Hand \& Finch, 2008). This type of equation was first derived by G.W. Hill to describe the effect of perturbations on the orbit of the Moon, and it occurs in many other places in physics, including the quantum motion of electrons in a periodic potential of a crystal (Hand \& Finch, 2008). The band theory of solids is based on a similar equation, as is the theory of propagating electromagnetic waves in a periodic structure (Hand \& Finch, 2008). Other applications include parametric amplifiers. Although $P(t)$ and $Q(t)$ terms are periodic in a Hill equation, in our case they are not. So, in our model, Eq. (11) is a Hill-like equation which has a dc source on its right side and a time-dependent damping coefficient (see Eq. 12) and a time-dependent spring coefficient (see Eq. 13) in terms of a damped forced oscillator model. The objective of Eq. (11) is to find the modifier function which can be then used to define the oscillation field (polarization response) of the material. Due to the time-dependent damping and spring coefficients, the modifier function is totally coupled with the time dependency or time profile of the applied field.

Eq. (6) could also have been solved directly in the temporal domain, in which case we would have lost the analogy with the Hill-like equation. But the appropriateness of using the more complicated approach with the modifier function has solid physical reasons. In the case of a USCP excitation, the polarization response of the material is not unique all through the pulse continuance. Due to the shortness of the duration of the applied USCP comparing to the relaxation time of the bound electron, the interaction dynamics and the ability of the material to sense and follow the applied USCP field during its continuance will be completely different than the conventional matter-field interaction approach. In Eq. (6), physical parameters (damping and spring coefficients) are constant. However, the interaction dynamics will not be constant during the USCP excitation. So, in order to penetrate the effect of the applied field into the oscillator model via these physical parameters to have a better understanding of the oscillation response of the material under USCP excitation, we must find the definition of these physical parameters in terms of the applied field and the physical constants of the system (material). Eq. (12) and Eq. (13) are these definitions. They are being used in Eq. (11) to find the modifier function which has been embedded into Eq. (6). The physical dimension of the modifier function is a dipole moment per unit force. It frames the time dependency and the phase delay of the oscillation field of the bound electron under USCP excitation.

\subsection{Numerical results and discussions for first case assumptions}

In Fig. 4, different interaction characteristics of Laguerre and Hermitian pulses are shown for a fixed, relatively low value of damping constant $\left(\delta_{o}=1 \times 10^{14} \mathrm{~Hz}\right)$. Due to the definition: 

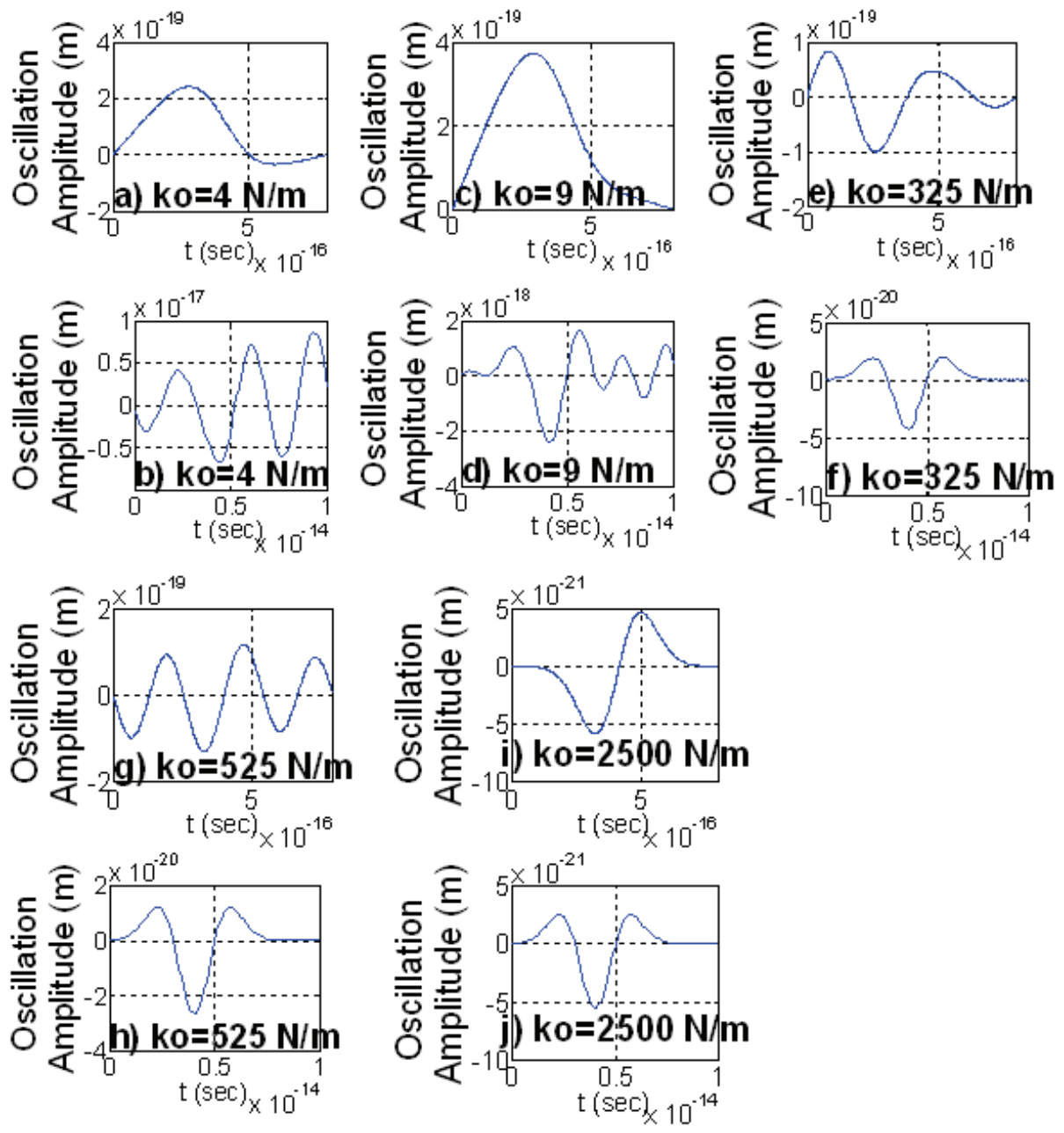

Fig. 4. Bounded electron motion under Laguerre USCP excitation ((a), (c), (e), (g), (i)) and Hermitian USCP excitation ((b), (d), (f), (h), (j)) for various values of spring constant $\left(k_{o}\right)$ with a fixed damping constant $\left(\delta_{o}=1 \times 10^{14} \mathrm{~Hz}\right)$.

$w_{0}=\sqrt{k_{o} / m_{e}},\left(m_{e}\right.$ is the mass of electron, $k_{o}$ is the spring constant for bound electron), the free oscillation frequency of material is in UV range for spring constant values of $4 \mathrm{~N} / \mathrm{m}, 9$ $\mathrm{N} / \mathrm{m}, 325 \mathrm{~N} / \mathrm{m}, 525 \mathrm{~N} / \mathrm{m}$ [Figs. 4(a), 4(b), 4(c), 4(d), 4(e), 4(f), 4(g), 4(h)], $650 \mathrm{~N} / \mathrm{m}$ [Fig. 8(b)] and $750 \mathrm{~N} / \mathrm{m}$ [Figs. 5(b), 8(c)]. For spring constant values of $1500 \mathrm{~N} / \mathrm{m}$ [Fig. 5(c)], $2500 \mathrm{~N} / \mathrm{m}$ [Figs. 4(i), 4(j)] and $7500 \mathrm{~N} / \mathrm{m}$ [Fig. 8(d)], the free oscillation frequency is in X-ray range. As it is clearly seen in Fig. 4, the Hermitian interaction has a more tendency to oscillation than the Laguerre interaction for relatively low values of spring constant [see Figs. 4(a), 4(b), 4(c), $4(\mathrm{~d})$ ]. As the spring constant is increased, Laguerre interaction gains a more oscillatory 
profile [see Figs. 4(e), 4(g)] while the oscillation due to the Hermitian pulse interaction stabilizes and its time profile settles down into the inverted phase time profile of the excitation pulse (inverted Mexican Hat) [see Figs. 4(f), 4(h), 4(j)]. Here, the amplitude of oscillation or the amplitude of trembling-like motion of the electron is in the range of $10^{-20} \mathrm{~m}$ $-10^{-21} \mathrm{~m}$ which is in the scale of electron radius length. Finally, as the spring constant is increased to relatively higher values, the Laguerre interaction settles down into the inverted phase time profile of the excitation pulse, too (inverted Laguerre pulse) [see Fig. 4(i)]. Fig. 4 shows a very clear distinction between the interaction characteristics of Laguerre and Hermitian USCPs until the spring constant is $2500 \mathrm{~N} / \mathrm{m}$ (after this value, we obtain only the inverted phase time profile of the excitation source for the oscillation). The oscillation characteristics of bound electron under different single USCP sources originates from modifier function approach. The Hill-like equation, which is the result of the modification on the classic Lorentz damped oscillator model with the modifier function approach, causes the time varying physical parameters to come into play during the interaction process. Since these physical parameters (time varying damping and spring coefficients) are absolutely source dependent, they behave differently in the pulse duration of each different USCP source. As a result of this, we see different oscillation profiles for a bound electron under a single Laguerre and Hermitian USCP excitations.
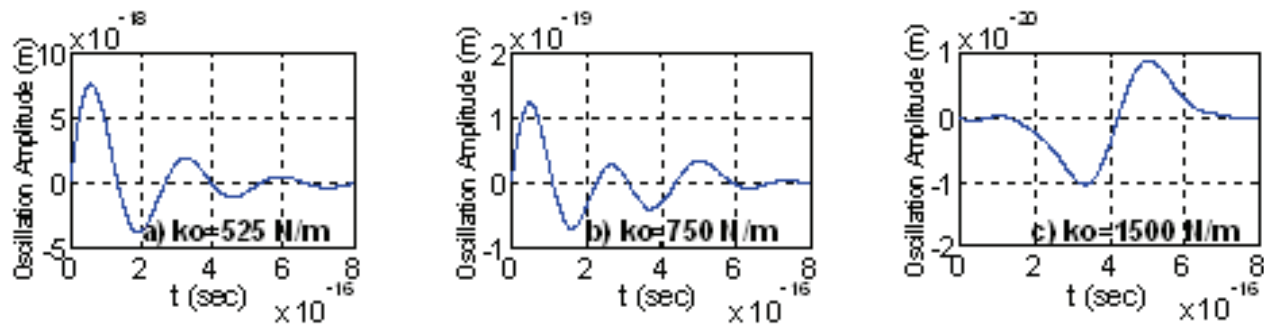

Fig. 5. Laguerre pulse excitation oscillations for damping constant: $\delta_{o}=1 \times 10^{16} \mathrm{~Hz}$.

In Fig. 5, response of a bound electron is shown for a Laguerre pulse excitation for varying values of spring constant with a fixed, relatively higher damping constant value $\left(1 \times 10^{16}\right)$ than the previous case (Fig.4). An interesting feature here in Fig. 5(a) and Fig. 4(g) is that although they are at the same spring constant value, they show different oscillation characteristics. Due to a higher dampimg coefficient in Fig. 5(a), while the oscillation attenuates quicker at the second half cycle of the Laguerre USCP than in Fig. 4(g), it hits to a higher peak at the first half cycle of the excitation pulse than in Fig. 4(g). So, for a reasonable value of spring constant, while relatively higher damping coefficient makes the first half cycle of the Laguerre USCP more efficient in the means of interaction, it makes the second half cycle less efficient. In order to compare oscillation results more detailly between Figs. $5(\mathrm{a})$ and $4(\mathrm{~g})$, it is necessary to look at their physical parameter solutions such as time varying damping and time varying spring coefficients. As it is explained above, these time varying parameters come into play due to the nature of "Modifier Function Approach". In Fig. 6, time varying damping coefficient, time varying spring coefficient and the modifier function solutions of 

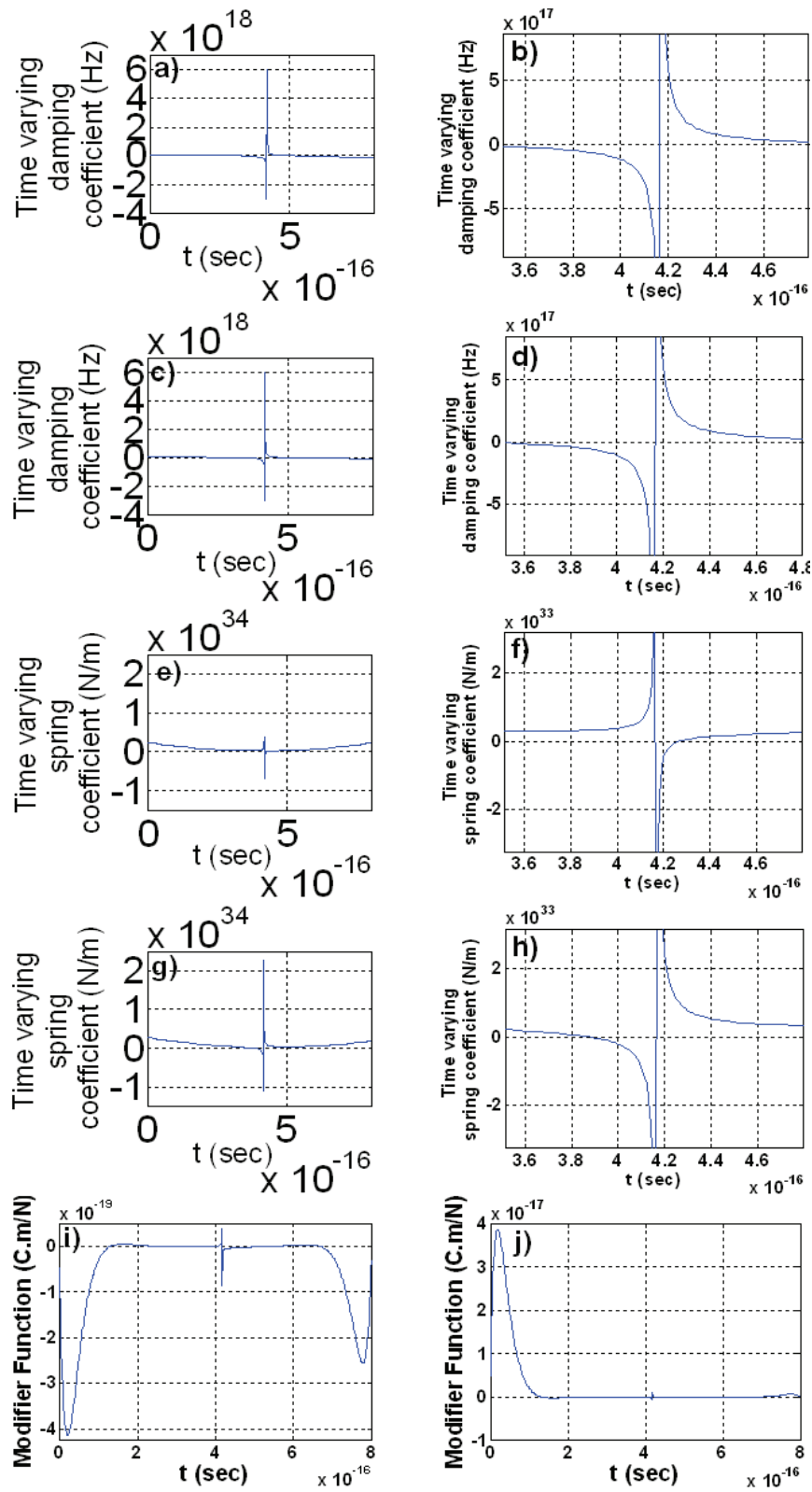

Fig. 6. Laguerre pulse excitation physical parameter solutions for spring constant $k_{o}=525 \mathrm{~N} / \mathrm{m}$. (a), (b), (e), (f) and (i) are the solutions of Fig. 4(g) (damping constant $\delta_{o}=1 \times 10^{14} \mathrm{~Hz}$ ). (b) and (f) are the magnified views of (a) and (e) respectively. (c), (d), (g), (h) and (j) are the solutions of Fig. 5(a) (damping constant $\delta_{o}=1 \times 10^{16} \mathrm{~Hz}$ ). (d) and (h) are the magnified views of $(\mathrm{c})$ and $(\mathrm{g})$ respectively. 

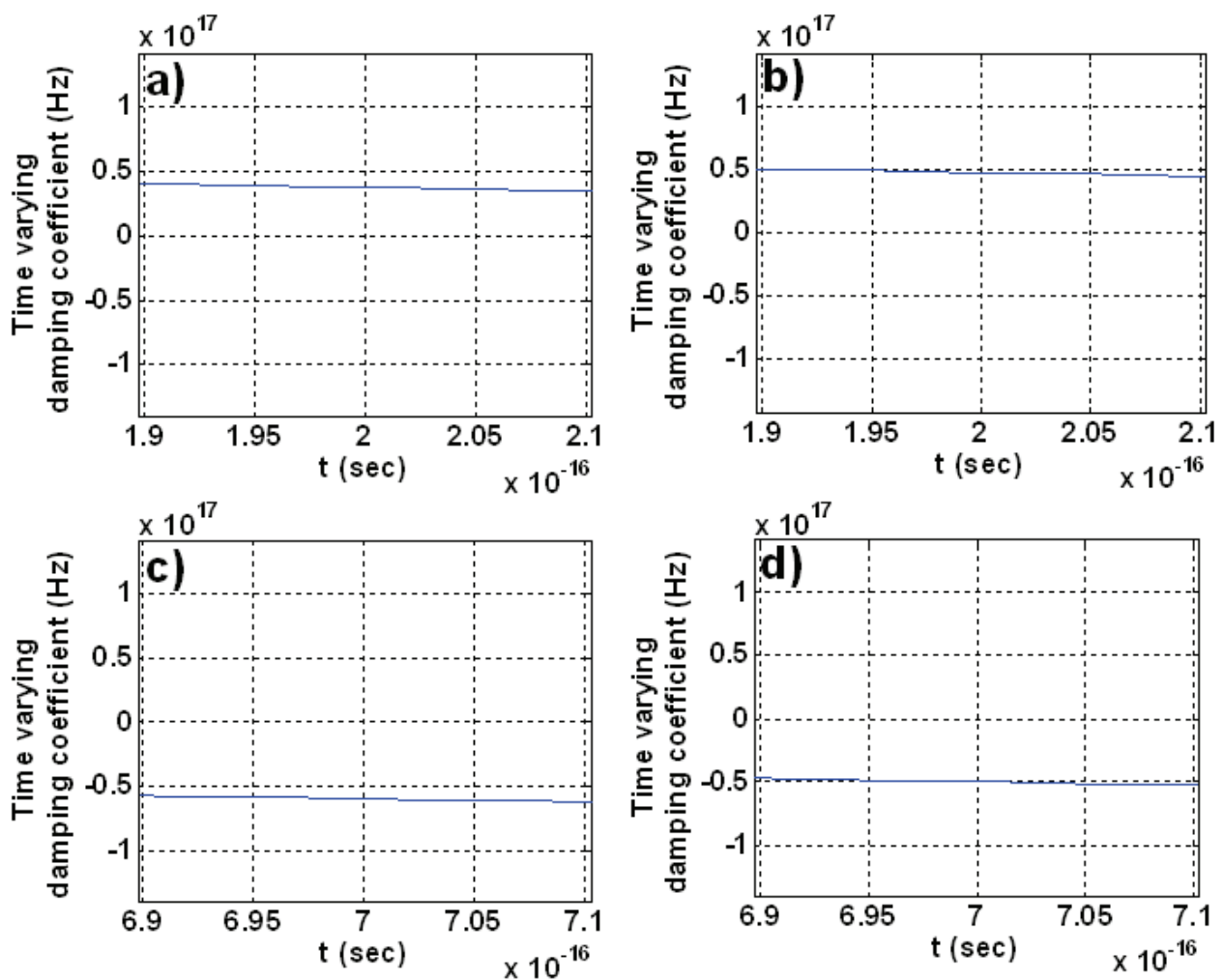

Fig. 7. (a) - (b): Magnified views of left wings of Figs. 6(a)-6(c). (c) - (d): Magnified views of right wings of Figs. 6(a)-6(c).

Figs. 4(g) and 5(a) are shown respectively for two different damping constant values with a fixed spring constant at $525 \mathrm{~N} / \mathrm{m}$. In Figs. 6(a) and 6(c), a sudden jump is seen in the time varying damping coefficient profiles at the time point where the excitation pulse changes its polarization direction. Although they look identical, the magnified views [see Figs. 7(a), 7(b), 7(c), 7(d)] of the left and right wings of the damping coefficient show the difference between two different damping constant cases. Here, the left wing corresponds to the first half cycle, right wing corresponds to the second half cycle of the Laguerre excitation pulse. Comparing the amount of the change on the y-axis with the time duration on the $x$-axis between Figs. 7(a) - 7(b), and 7(c) - 7(d), it is easy to see the reasonable amount of difference to affect the solution of modifier function [see Figs. 6(i), 6(j)]. For time varying spring coefficients [see Figs. 6(e), 6(g)], a significant difference is seen in the time profile although the spring constant values are the same for both cases. The jump in Fig. 6(g) hits a higher peak than the jump in Fig. 6(e). This can be a reasonable explanation for a relatively low oscillation tendency in the second half cycle of Fig. 5(a) than the Fig. 4(g). It can be said that, due to the dissipation of higher energy, this jump causes a lower oscillation profile for the bound electron during its interaction with the second half cycle of the Laguerre pulse in Fig. 5(a) than in Fig. 4(g). In Fig. 5(c), as the spring constant is increased to a relatively higher values, same as in Fig. 4(i), the oscillation profile settles down into the inverted time phase 
profile of the excitation pulse. Different from Fig. 4(i), the oscillation settles down at a relativley lower spring constant value. So, it can be said that, for a higher damping constant, a lower spring constant is enough to stabilize the oscillation profile in time domain.

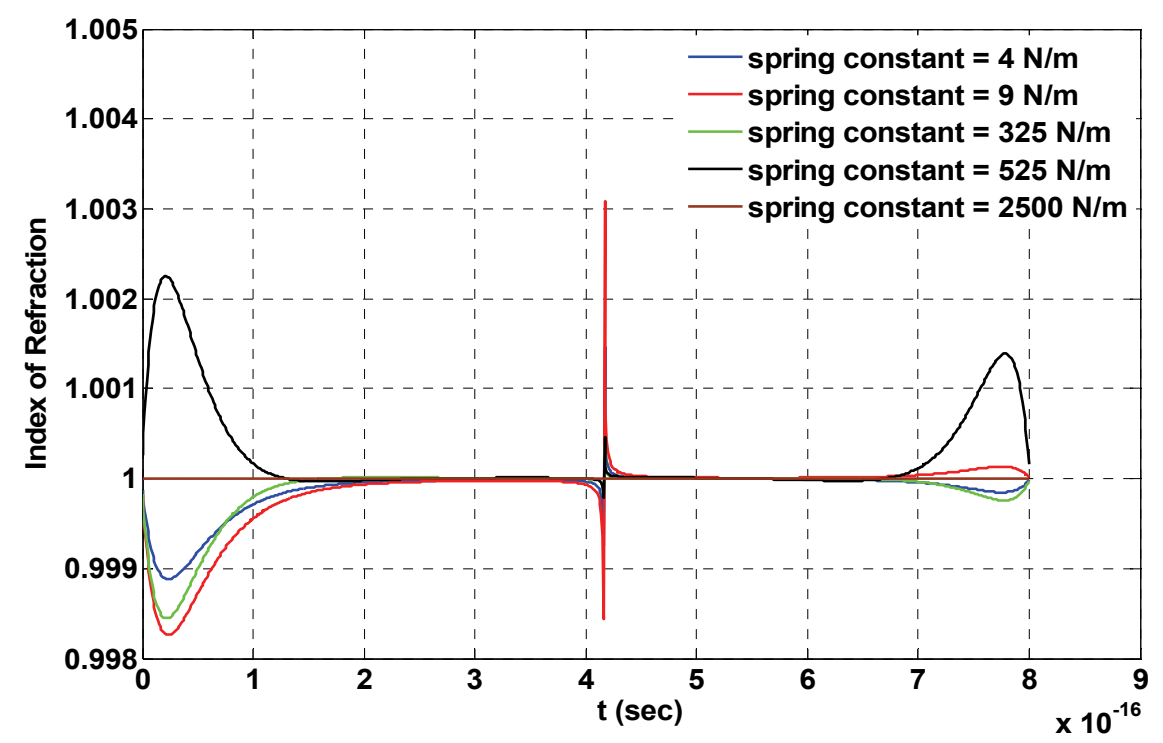

Fig. 8. Time dependent index of refraction during the interaction of a single Laguerre USCP with a bound electron without ionization for different spring constant values with a fixed damping constant value ( $\delta_{o}=1 \times 10^{14} \mathrm{~Hz}$ ). It is obtained from Eq. (4) where $P_{\text {pol }}(t)=-\operatorname{Nex}(t)$. Here $N=6.02 \times 10^{23}$ and $-e$ is the electron charge.

Fig. 8 shows the perturbation effect of an applied single Laguerre USCP on the index of refraction during its continuance for varying spring constants with a fixed damping constant value. As it is clearly seen in Fig. 8, for all spring constant values except the relatively higher case $(2500 \mathrm{~N} / \mathrm{m})$, there are three regions where the perturbation effects are dominant. These are the trailing and leading regions of the pulse and the time region where the applied electric field changes its polarization sign. The change in the index of refraction around the trailing and leading edges is not as sharp as the change at the point where the polarization sign of the field changes. To see this sudden effect more clearly, the zoomed view of this region is shown in Fig. 9.

The same type of perturbation behavior seen in Fig. 8, is seen in the interaction of a single Hermitian USCP with a bound electron, too (see Fig. 10). Both of these figures have the same damping constant value. The only difference in the time dependent perturbation of index of refraction between these two cases is that since there are two points where the Hermitain USCP field changes its polarization sigh, we have sudden changes in the perturbation of index of refraction twice around these point. The zoomed view of these regions shows the sudden effects more clearly in Fig 11.

In Fig. 12, we see a similar type of change in the time dependent index of refraction for damping constant $\delta_{o}=1 \times 10^{16} \mathrm{~Hz}$. 


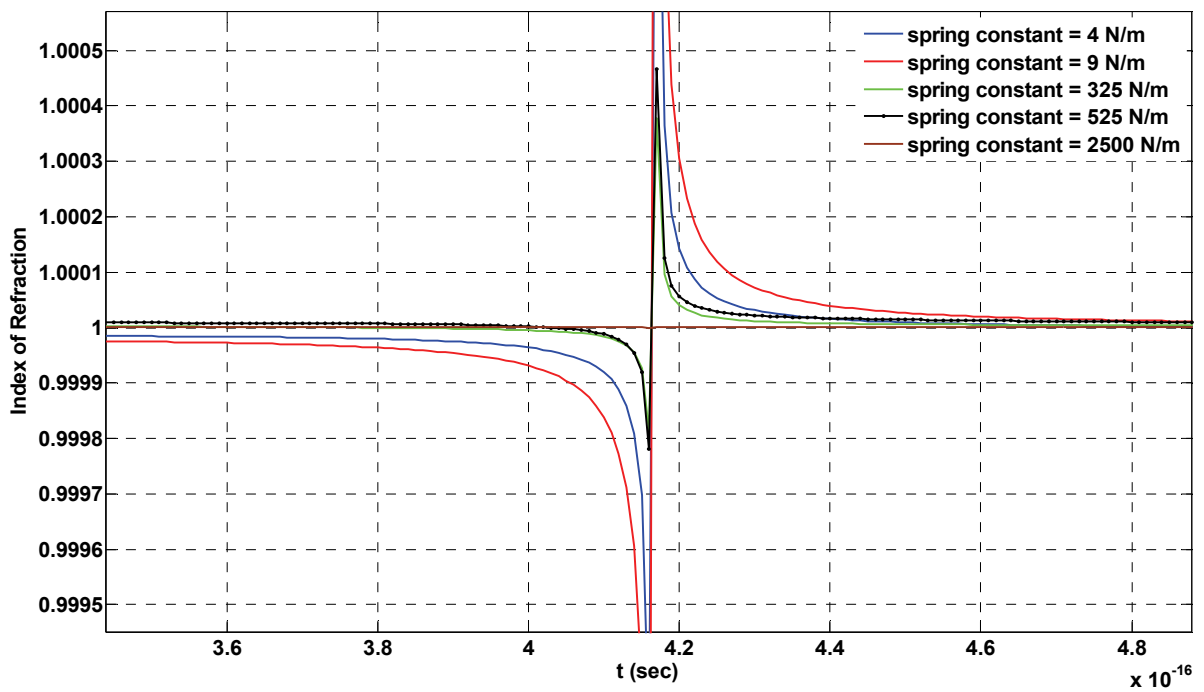

Fig. 9. The jump in the time dependent index of refraction where the electric field changes its polarization sign.

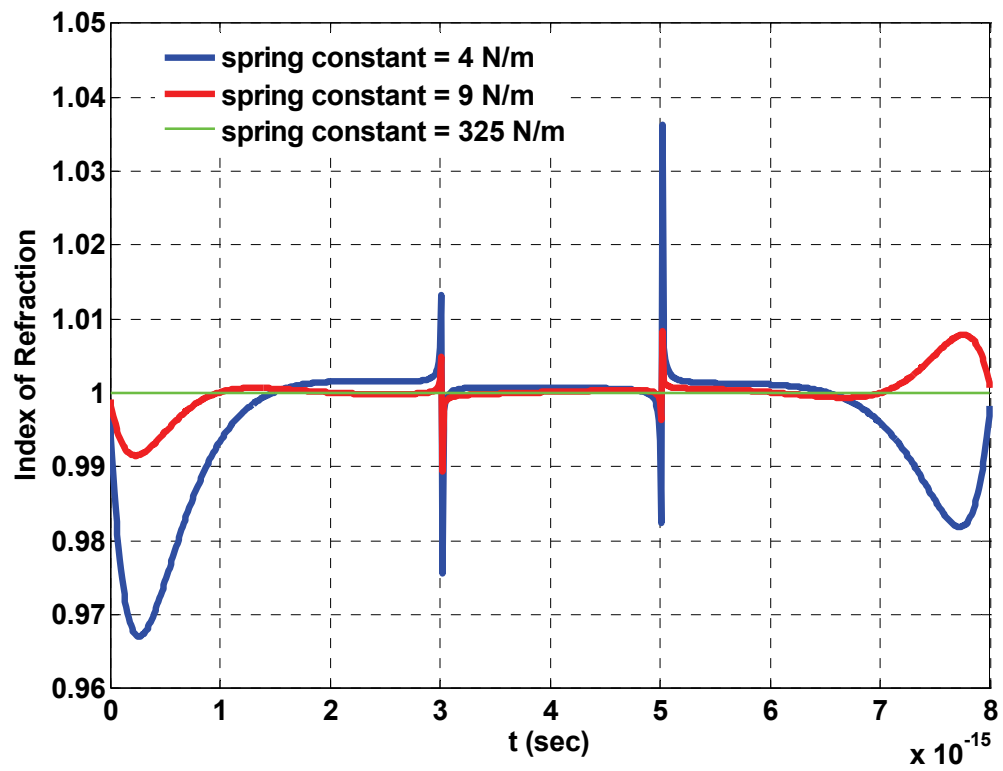

Fig. 10. Time dependent index of refraction during the interaction of a single Hermitian USCP with a bound electron without ionization for different spring constant values with a fixed damping constant value $\left(\delta_{o}=1 \times 10^{14} \mathrm{~Hz}\right)$. 


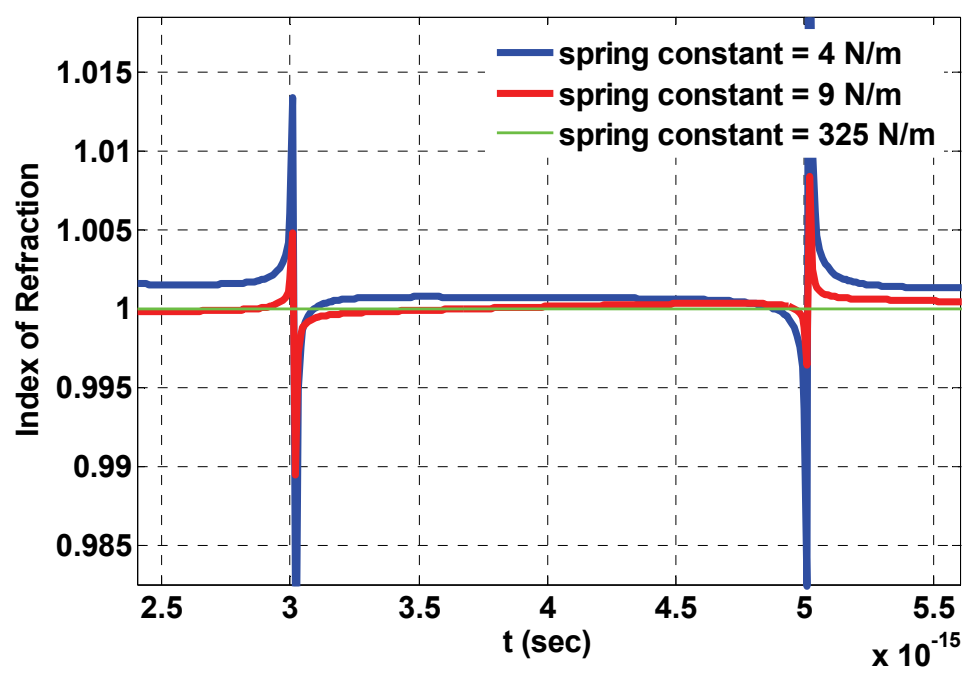

Fig. 11. The jump in the time dependent index of refraction where the electric field changes its polarization sign for single Hermitian USCP interaction.

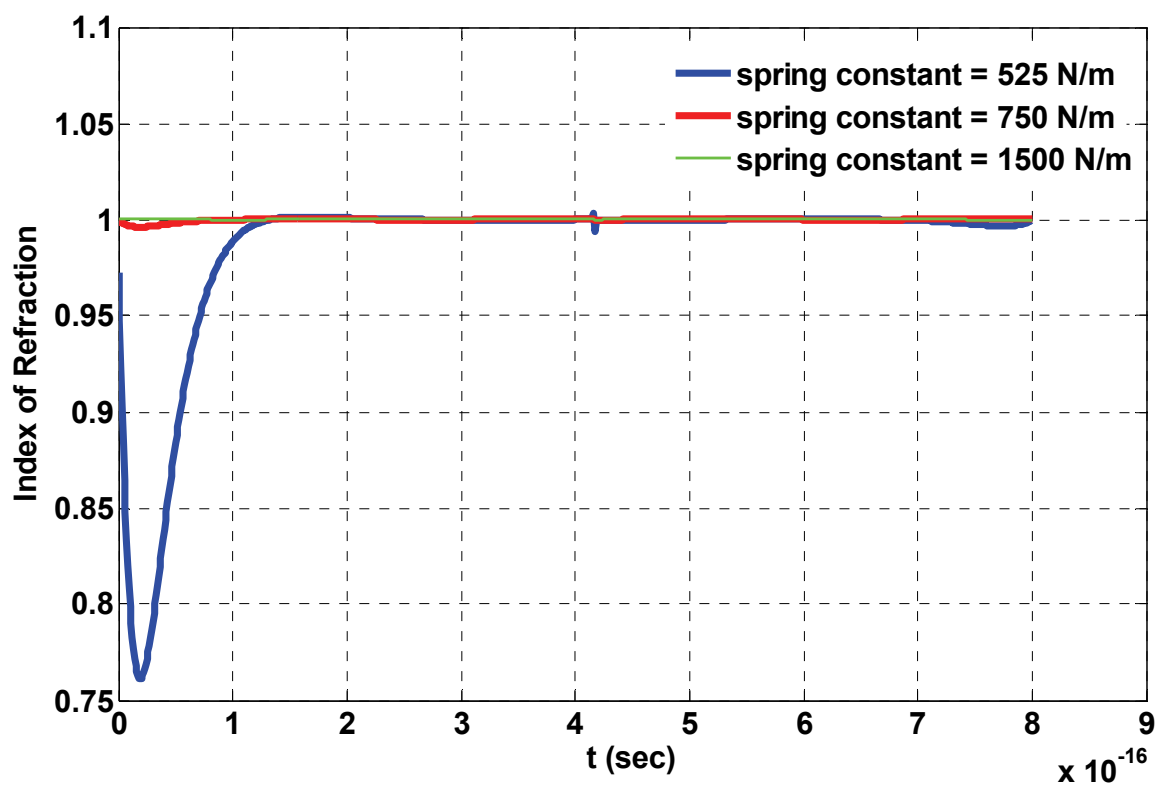

Fig. 12. Time dependent index of refraction during the interaction of a single Laguerre USCP with a bound electron without ionization for different spring constant values with a fixed damping constant value $\left(\delta_{o}=1 \times 10^{16} \mathrm{~Hz}\right)$. 

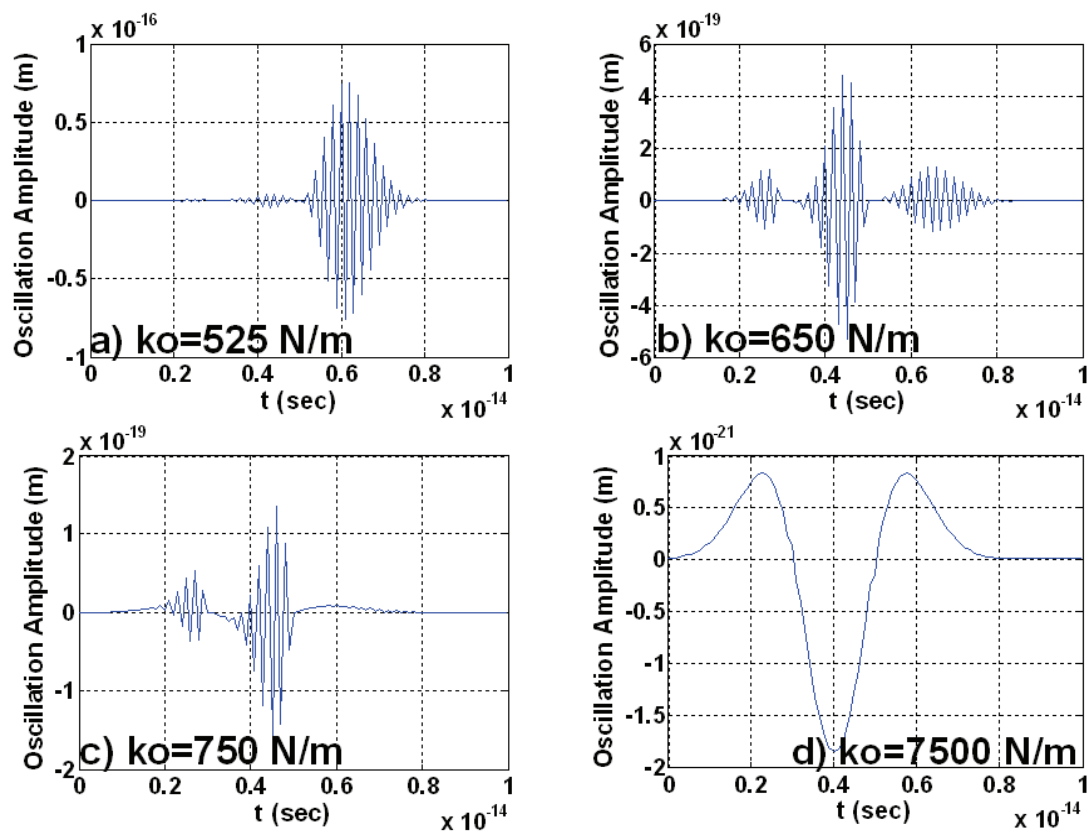

Fig. 13. Hermitian pulse excitation oscillations for damping constant: $\delta_{o}=1 \times 10^{17} \mathrm{~Hz}$

For a damping constant value of $1 \times 10^{17}$ (Fig. 13), very different oscillation behaviors are seen than the previous cases (Fig. 4) of Hermitian pulse excitation. The most prominent feature in Figs. 13(a), 13(b) and 13(c) is the high frequency oscillation profile with a phase delay with respect to the excitation pulse. In Fig. 13, spring constant is increased gradually from 13(a) to 13(c) while keeping the damping value constant. For a relatively low value of spring constant in Fig. 13(a), the main lobe and the trailing tail of the excitation pulse have almost no effect on the oscillation of the electron. The bound electron starts sensing the leading tail of the Hermitian excitation after a phase delay of 5 fs. In Fig. 14, the modifier function solutions for the Hermitian pulse excitation for Fig. 13 are shown. As it is clearly seen in Fig. 14(a), modifier function suppresses the interaction effect of main lobe and the trailing tail of Hermitian function. As a result of this, the bound electron starts sensing the excitation pulse with a phase delay [Fig. 13(a)] associated with the modifier function. Same behaviour of the modifier function is seen in Figs. 14(b) and 14(c), too. As a result of this, approximately 2 fs phase delay occurs in Figs. 13(b) and 13(c). In Fig. 14(d), the type of modifier function is seen that gives a completely phase inverted time profile of the excitation pulse for the oscillation of the bound electron. In Fig. 13(d), the stabilized oscillation profile is seen as a result of this modifier function.

In Fig. 15, as in the Fig. 13, there is a high oscillation frequency behaviour in the perturbation effect of the single Hermitian USCP on the index of refraction. Especially, the magnitude of the perturbation effect is more significant around the main lobe and the trailing edge regions than the leading edge region of the applied field. The effect of the Hermitian USCP on the index of refraction decreases as the spring constant increases for the given fixed damping constant value. 

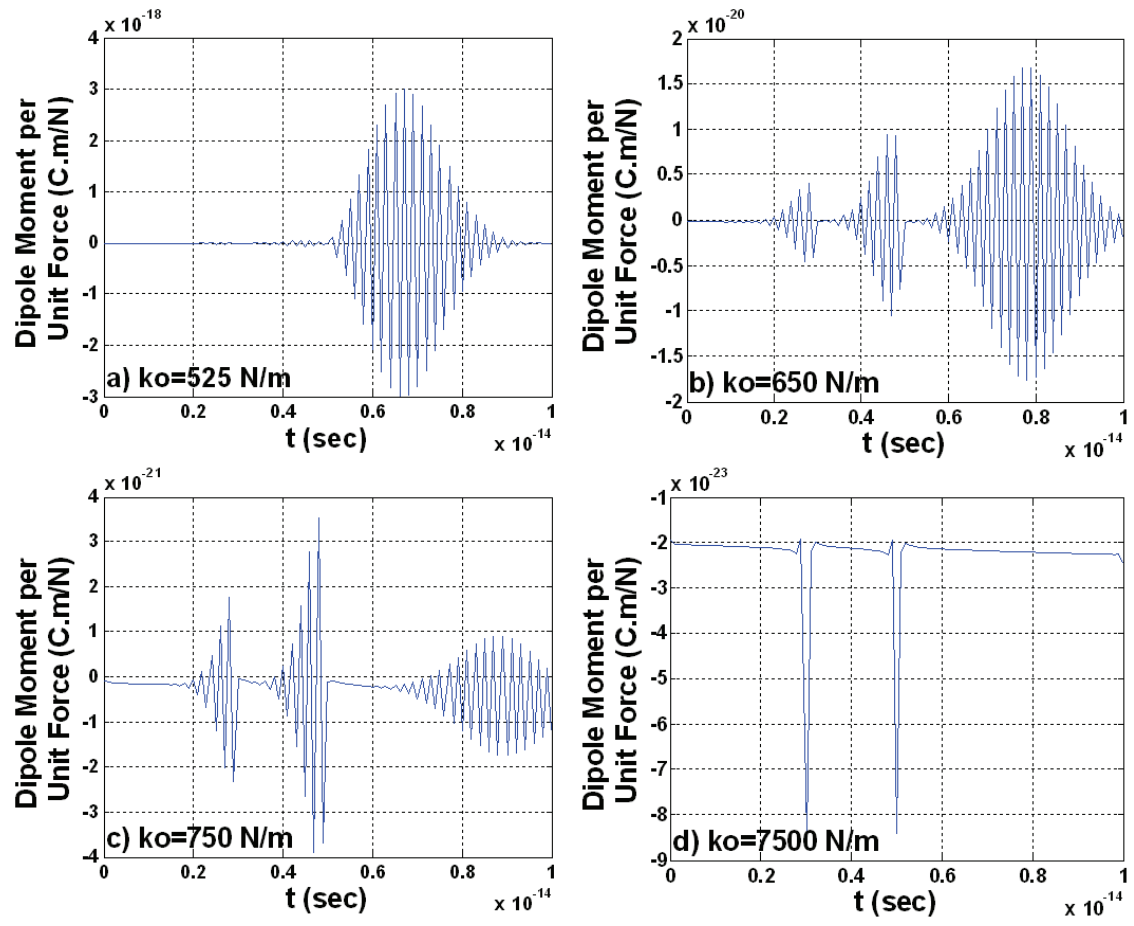

Fig. 14. Hermitian pulse excitation modifier functions for damping constant: $\delta_{o}=1 \times 10^{17} \mathrm{~Hz}$.

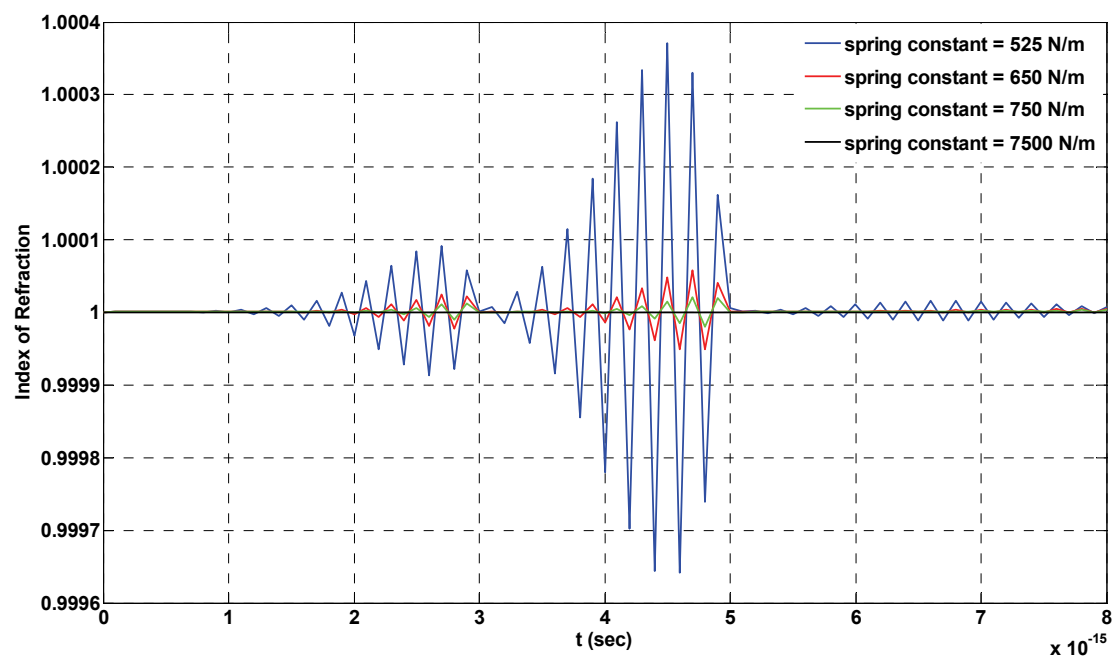

Fig. 15. Time dependent index of refraction during the interaction of a single Hermitian USCP with a bound electron without ionization for different spring constant values with a fixed damping constant value $\left(\delta_{o}=1 \times 10^{17} \mathrm{~Hz}\right)$. 


\subsection{Mathematical model for convolutional modifier function approach}

In section 1.2, we explained why the oscillation field of the bound electron under single USCP exposure must be defined in terms of the multiplication of the applied USCP with a modifier function. In a more realistic approximation, we need to include a constant updating between the electron motion and the time dependent applied field. This is the major difference between approaches used in sections 1.2 and 2.1. Suppose that we are applying two different USCPs ranging in different spectral content on to the same type of material at different points. If we assume that the majority of the spectral content of one of these USCPs is relatively closer to the natural oscillation frequency of the bound electron of the material than the spectral content of the other USCP (see Fig. 16), then it will not be realistic to consider exactly the same type of time domain USCP interaction mechanism (modifier function approach that has been explained in section 1.2) for both of these two different USCPs.

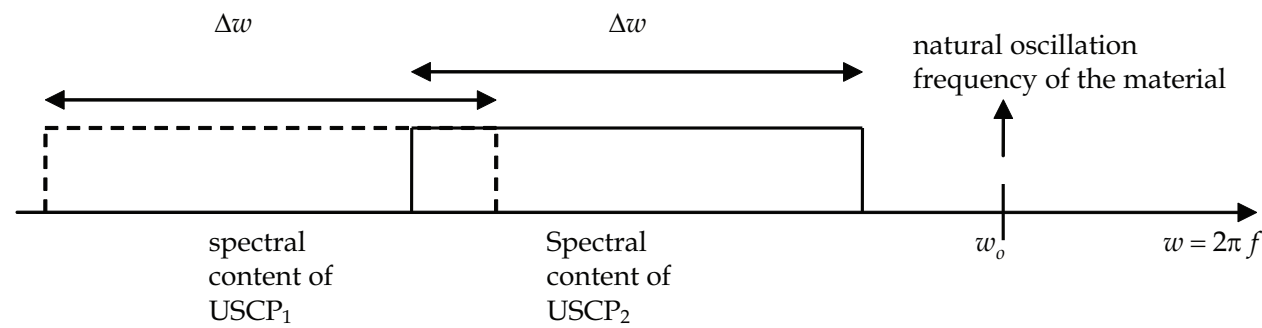

Fig. 16. Spectral content of two different USCPs with the same pulse duration. They are being applied to different points on a material which has a natural oscillation frequency of $w_{0}$.

As it is seen in Fig. 16, we note that since the majority of the spectral content of $\mathrm{USCP}_{2}$ is closer to $w_{0}$ than the majority of the spectral content of $\mathrm{USCP}_{1}$, in the context of interaction efficiency, the interaction of $\mathrm{USCP}_{2}$ will be relatively more intense than the interaction of $\mathrm{USCP}_{1}$ for the given spectral content and for the given natural oscillation frequency. Given the formulation provided in section 1.2, we are just directly masking (multiplying) the modifier function (that we found from Eq. 11) on to the time domain profile of $\mathrm{USCP}_{1}$ to find the oscillation field of the bound electron during the continuance of this pulse. If we follow the same procedure to calculate the oscillation field of the bound electron under $\mathrm{USCP}_{2}$ excitation, this will cause us to miss the cumulative tendency due to the memory effect of the oscillation field of the bound electron in time domain due to the interaction with single $\mathrm{USCP}_{2}$ compared to the interaction with single $\mathrm{USCP}_{1}$. In order to take into consideration the cumulativeness effect under $\mathrm{USCP}_{2}$ excitation, instead of defining oscillation as in Eq. 7, we need to define the time dependent electron motion with a convolution operation: since a convolution can be considered as an operation that shows the effect of current and past inputs to the current output of a system:

$$
x(t)=x_{o}(t) * E(t) .
$$

If we plug Eq. (14) into Eq. (6), we obtain

$$
\frac{d^{2}}{d t^{2}}\left(x_{o}(t) * E(t)\right)+\gamma_{o} \frac{d}{d t}\left(x_{o}(t) * E(t)\right)+\frac{k_{o}}{m_{e}}\left(x_{o}(t) * E(t)\right)=\frac{e}{m_{e}} E(t) .
$$


Eq. 15 allows us to obtain the oscillation field after the pulse (wake-field) due to the nature of convolution operation in Eq. 14. The modifier function is a hidden function that must be evaluated first to find the oscillation field caused by the USCP excitation where the source duration is much shorter than the relaxation dynamics of the material. Due to the nature of convolution operation in Eq. 14, although the USCP actually vanishes at $t=\tau$ (where $\tau$ is the pulse duration), the modifier function will still exist after the end of the pulse and our technique evaluates the oscillation field after the pulse duration due to the memory effect of the convolution operation.

In order to find the modifier function in Eq. 15, different mathematical solution techniques can be used. For the work in this book chapter, let us use Eq. 14 in the following form:

$$
\begin{gathered}
f(t)=x_{o}(t)+x_{o}(t) * E(\tau), \\
f(t)=x_{o}(t)+\int_{0}^{t} x_{o}(t-\tau) E(\tau) d \tau,
\end{gathered}
$$

which is called Volterra Integral Equation (VIE) of the second kind where the source function $f(t)$ and the kernel function $E(\tau)$ are given and $x_{o}(t)$ is the unknown function. There are many existing state of the art numerical techniques for solving the VIE in the literature (e.g., Tang et al., 2008). Future work will be undertaken to solve this equation following numerical techniques developed specifically for the approximate solution of VIE. In future work it is anticipated that publications will compare numerical solutions to our simpler mathematical solution approach. However, in this book Chapter we will follow a simpler mathematical procedure in order to obtain physical understanding and insight of differences between convolutional modifier function approach (section 2.1) and the modifier function approach used in section 1.2. Let's define the convolution integral in Eq. 17 as:

$$
\int_{0}^{t} x_{o}(t-\tau) E(\tau) d \tau=f(t)-x_{o}(t)
$$

where $f(t)$ is going to be a reasonable trial function that will be defined for finding the modifier function in Eq. 15. By plugging the definition in Eq. 18 into Eq. 15, we obtain:

$$
\frac{d^{2}}{d t^{2}} x_{o}(t)+\gamma_{o} \frac{d}{d t} x_{o}(t)+\frac{k_{o}}{m_{e}} x_{o}(t)=F(t) \text {, }
$$

where

$$
F(t)=\frac{d^{2}}{d t^{2}} f(t)+\gamma_{o} \frac{d}{d t} f(t)+\frac{k_{o}}{m_{e}} f(t)-\frac{e}{m_{e}} E(t)
$$

While in Eq. 11 in section 1.2 we are calculating the modifier function for time dependent damping and spring coefficients, in Eq. 19 we calculate the modifier function for constant damping and spring coefficients with a time dependent source term modified by the trial function $f(t)$. This approach allows us to incorporate the cumulative tendency of the oscillation field and memory effect originating from the spectral content of the USCP and to have constant damping and spring coefficients during the pulse continuance. 


\subsection{Numerical results for second case}

For our numerical calculations, we used the following forms as two trial functions simultaneously for the Laguerre USCP excitation case:

$$
\begin{aligned}
& f_{1}(t)=f_{o 1}\left(\exp \left(-\beta^{2}\right)-\operatorname{Sin}\left(\beta^{a}\right)\right), \\
& f_{2}(t)=f_{o 2}\left(\exp \left(-\beta^{2}\right)+\operatorname{Sin}\left(\beta^{a}\right)\right),
\end{aligned}
$$

where $a$ is ranging from 1 to $\mathrm{n}$ and $\beta=t-z c^{-1} / t_{0}$. So, at the end of the calculations, the total oscillation field has been evaluated as:

$$
x(t)=\frac{1}{2 n} \sum_{i=1}^{n}\left[x_{o 1 i}(t) * E(t)+x_{o 2 i}(t) * E(t)\right],
$$

where $x_{o 1 i}(t)$ is calculated for $f_{1}(t)_{a=i}$ and $x_{o 2 i}(t)$ is calculated for $f_{2}(t)_{a=i}$ from Eq. 19. For the Hermitian USCP excitation case, we used the following form as the trial function in the numerical calculations:

$$
f_{3}(t)=f_{o 3}\left(1-\beta^{-a}\right)
$$

and the total oscillation field has been evaluated as:

$$
x(t)=\frac{1}{n} \sum_{i=1}^{n}\left[x_{o 3 i}(t) * E(t)\right],
$$

where $x_{o 3 i}(t)$ is calculated $f_{3}(t)_{a=i}$ from Eq. 19 .

The values of the amplitude constants $f_{01}, f_{02}$, and $f_{03}$ are dependent on the trial function and the number of trial functions that are chosen for the solution of the modifier function.

In Fig. 17, we see some important results of the convolutional modifier function approach on the oscillation field of the bound electron under Laguerre and Hermitian USCP excitation and both have close spectral content to the natural oscillation frequency of the material. Although there is not much difference in the oscillation frequency compared to the Fig. 4 in section 1.3, there is a significant difference in the oscillation amplitude where the convolutional modifier function approach has higher amplitudes. In addition to this (different than Fig. 4), in Fig. 17 we see some phase delay in the oscillation field with respect to the applied USCP for both Laguerre and Hermitian excitations (see Figs. 17(a), 17(b), 17(c), 17(e), 17(g), 17(h), 17(i) and 17(j)). Another significant result shown in Fig. 17, due to the nature of the convolution operation, we can see the oscillation in the wake-field after the continuance of the USCP.

For Fig. 18, we have a higher oscillation amplitude and almost the same oscillation frequency as compared to Fig. 5. Also in Figs. 18(a), 18(b) and 18(c) there is a phase delay which is not seen in Figs. 5(a) and 5(b). It is observed comparing Fig. 17 to Fig. 18 there is a significant difference in the wake-field oscillations which are attenuated much quicker in Fig. 18 after the end of the pulse continuance.

In Figs. 19 and 20, we plot the real and imaginary part of the perturbation effect of an applied single Laguerre USCP for the convolutional modifier function approach. The common behavior that we note in Figs. 8, 19 and 20 is that there is a sudden jump for real 
and imaginary parts of the index of refraction at the point where the USCP field changes its polarization sign. In comparison (see Fig. 8) the real part of the perturbation effect of the applied USCP vanishes at some regions of the Laguerre USCP as illustrated in Fig 19. As can be seen in Fig. 20, at the regions where the real part vanishes, the imaginary part of the perturbation effect on the index of refraction comes into play.
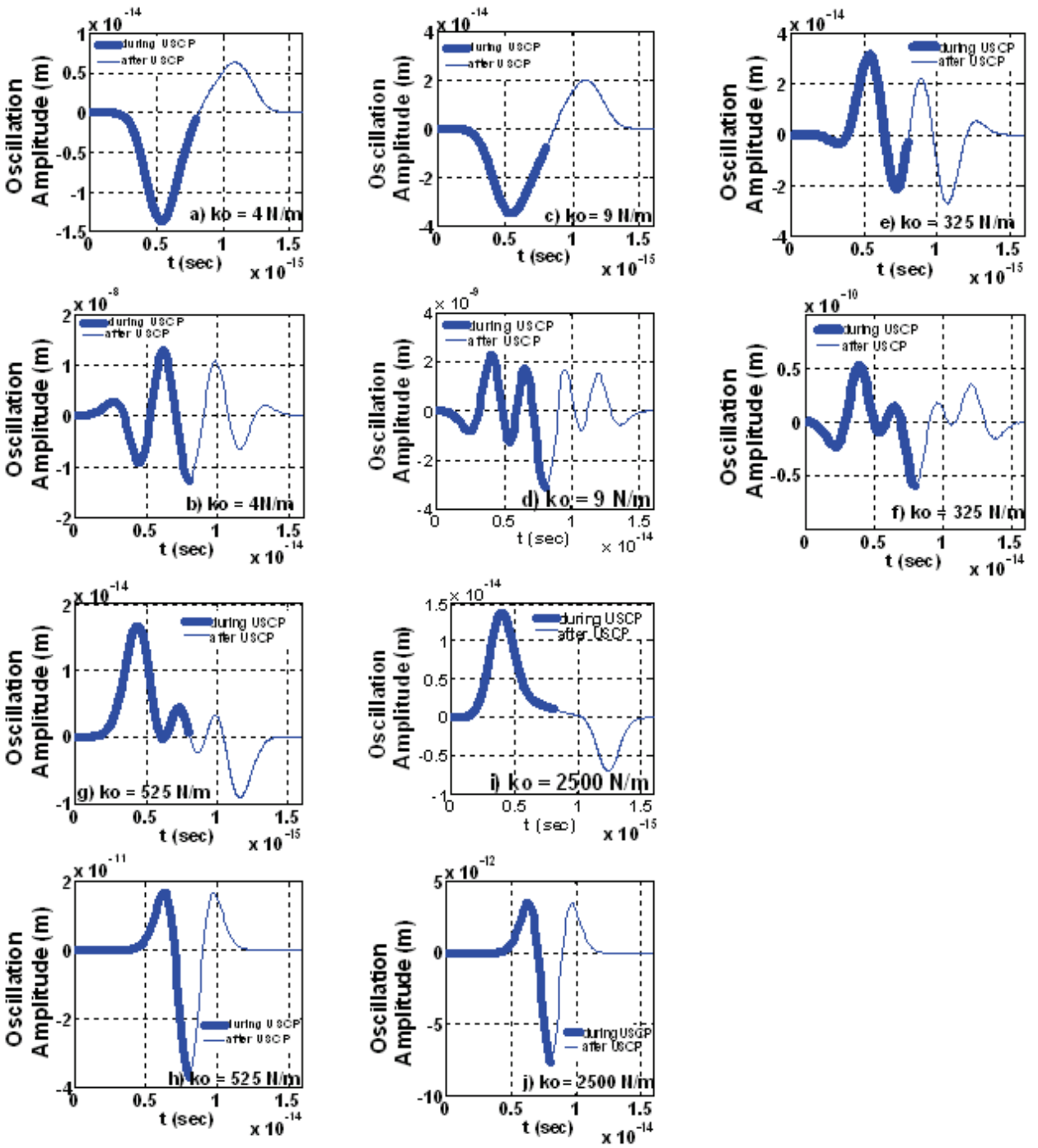

Fig. 17. Bounded electron motion for the convolutional modifier function approach under Laguerre USCP excitation ((a), (c), (e), (g), (i)) and Hermitian USCP excitation ((b), (d), (f), $(\mathrm{h}),(\mathrm{j}))$ for various values of spring constant $\left(k_{o}\right)$ with a fixed damping constant $\left(\delta_{o}=1 \times 10^{14} \mathrm{~Hz}\right)$. 

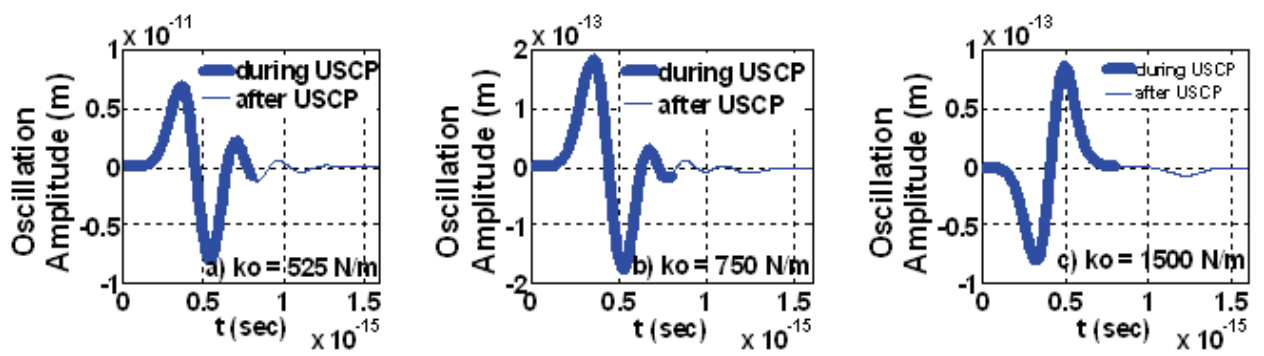

Fig. 18. Bounded electron motion for the convolutional modifier function approach under Laguerre USCP excitation for various values of spring constant $\left(k_{o}\right)$ with a fixed damping constant $\left(\delta_{o}=1 \times 10^{16} \mathrm{~Hz}\right)$.

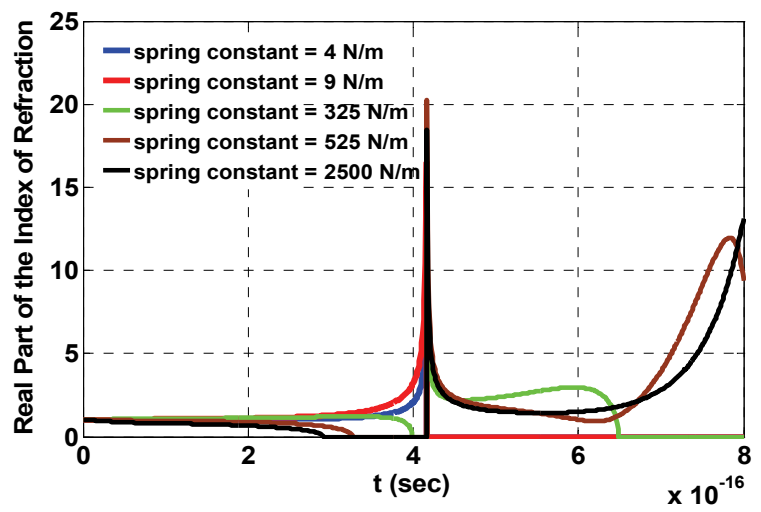

Fig. 19. Real part of the time dependent index of refraction during the interaction of a single Laguerre USCP with a bound electron without ionization for different spring constant values with a fixed damping constant $\left(\delta_{o}=1 \times 10^{14} \mathrm{~Hz}\right)$ [see Eq. 4].

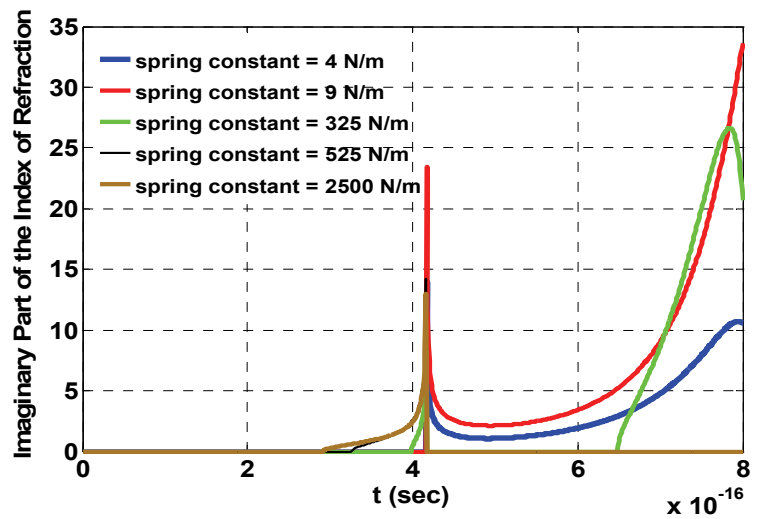

Fig. 20. Imaginary part of the time dependent index of refraction during the interaction of a single Laguerre USCP with a bound electron without ionization for different spring constant values with a fixed damping constant $\left(\delta_{o}=1 \times 10^{14} \mathrm{~Hz}\right)$ [see Eq. 4]. 


\section{Conclusion}

The results of this work indicate that if the applied field is a USCP, then it is not possible to separate the field into pieces to find the polarization effect of each part of the applied field on a bound electron since the USCP can not be further broken down into separate pieces of the applied field. The traditional Fourier method of multiplying the Delta function response with the applied field and integrating (superposing) this product in time can only be used for SVE approximation which is not realistic for single cycle pulses of unity femtosecond and attosecond applied fields. In a USCP case, the Lorentz oscillator model must be modified in order to find the polarization effect of a single USCP. Since a USCP is extremely broadband, it is not realistic to use a center frequency in the calculations as is done in the Fourier series expansion approach. Results in this work are presented on the transient response of the system during the USCP duration without switching to frequency domain. In order to accomplish this mathematically, we developed a new technique we label as the "Modifier Function Approach". The modifier function is embedded in the classic Lorentz damped oscillator model and by this way, we upgrade the oscillator model so that it is compatible with the USCP on its right side as the driving force. Results of this work also provide a new modified version of the Lorentz oscillator model for ultrafast optics. The results also indicate that the time response of the two models used to represent the USCP can alter the time dependent polarization of the material as it interacts with a single cycle pulse.

As a second model, we chose to provide a convolution of the applied field and the movement of the electron for a further refinement of the classical Lorentz damped oscillator model. The convolution approach allows one to incorporate previous motion of the electron with the interacting applied field. Results are compared for the motion of the electron for each case and the observed change in the index of refraction as a function of time for two different cases. As expected the index of refraction is not a constant in the ultra short time time domain under the assumptions applied in these studies. The motion of the electron is also highly dependent on the type of input single cycle pulse applied (Laguerre or Hermitian).

In future work, we plan on providing chirp to the pulse and performing the necessary calculations to show the motion of the electron and the effects on the index of refraction as a function of time.

\section{References}

Agrawal, G. P. Olsson, N. A. (1989). Self-phase modulation and spectral broadening of optical pulses in semiconductor laser amplifiers, IEEE Journal of Quantum Electronics, Vol. 25., No. 11., (November 1989).

Akimoto, K. (1996). Properties and Applications of ultrashort electromagnetic mono- and sub- cycle waves. Journal of the Physical Society of Japan, Vol. 65., No. 7., (2020-2032).

Blanc, S. P. Sauerbrey, R. Rae, S. C. Burnett, K. (1993). Spectral blue shifting of a femtosecond laser pulse propagating through a high-pressure gas, Journal of Optical Society of America, Vol. 10, No. 10, (October 1993).

Cole, K. S. Cole, R. H. (1941). Dispersion and absorption in dielectrics, Journal of Chemical Physics, Vol. 9., (341-351), (April 1941).

Corkum P. B. (200). Attosecond science Nature Physics 3, (381 - 387).

a. Couairon, A. Biegert, J. Hauri, C. P. Kornelis, W. Helbing, F. W. Keller, U. Mysyrowicz, A. (2006). Self-compression of ultrashort laser pulses down to one optical cycle by filamentation, Journal of Modern Optics, Vol. 53., No. 1-2., (January 2006). 
b. Couairon, A. Biegert, J. Hauri, C. P. Kornelis, W. Helbing, F. W. Keller, U. Mysyrowicz, A. (2006). Self-compression of ultra-short laser pulses down to one optical cycle by filamentation, Journal of Modern Optics, Vol. 53., Issue 1\&2., (January 2006), (75 - 85).

Crisp, M. D. (1970). Propagation of small-area pulses of coherent light through a resonant medium. Physical Review A, Vol. 1., No. 6., (June 1970).

Daniel, V. V. (1967). Dielectric Relaxation. Academic Press, New York.

Djurisic, A. B. Li, E. H. (1998). Modeling the index of refraction of insulating solids with a modified Lorentz oscillator model. Applied Optics, Vol. 37., No. 22., (August 1998).

Dvorak, S. L. Dudley, D. G. (1995). Propagation of ultrawideband electromagnetic pulses through dispersive media. IEEE Transaction of Electromagnetic Compatibility, Vol. 37., No. 2., May 1995.

Eloy, J. F. Moriamez, F. (1992). Spectral analysis of EM ultrashort pulses at coherence limit. Modelling. SPIE Intense Microwave and Particle Beams III, Vol. 1629.

Eloy, J. F. Wilhelmsson, H. (1997). Response of a bounded plasma to ultrashort pulse excitation. Physica Scripta, Vol. 55., (475-477).

Gutman, A. L. (1998). Electrodynamics of short pulses for pulse durations comparable to relaxation times of a medium. Doklady Physics, Vol. 43., No. 6, 1998., (343-345).

Gutman, A. L. (1999). Passage of short pulse throughout oscillating circuit with dielectric in condenser. Ultra-Wideband, Short-Pulse Electromagnetics 4, Kluwer Academic / Plenum Publishers, New York.

Hand, L. N. Finch, J. D. (2008). Analytical Mechanics. Cambridge University Press, $7^{\text {th }}$ edition, Cambridge.

Hovhannisyan, D. (2003). Propagation of a femtosecond laser pulse of a few optical oscillations in a uniaxial crystal. Microwave and Optical Technology Letters, Vol. 36., No. 4., (February 2003).

Itatani, J. Levesque, J. Zeidler, D. Niikura, H. Pépin, H. Kieffer, J. C. Corkum, P. B. Villeneuve, D. M. (2004). Tomographic imaging of molecular orbitals. Nature 432, (867-871).

Joseph, R. M. Hagness, S. C. Taflove, A. (1991). Direct time integral of Maxwell's equations in linear dispersive media with absorption for scattering and propagation of femtosecond electromagnetic pulses. Optics Letters, Vol. 16., No. 18., (September 1991).

Kinsler, P. New, G. H. C. (2003). Few-cycle pulse propagation. Physical Review A 67, 023813

Kozlov S. A., Sazanov S. V. (1997). Nonlinear propagation of optical pulses of a few oscillations duration in dielectric media. JETP 84 (2), (February 1997).

Krauss, G. Lohss, S. Hanke, T. Sell, A. Eggert, S. Huber, R. Leitenstorfer, A. (2009). Synthesis of a single cycle of light with compact erbium-doped fibre technology. Nature Photonics 4., (33-36).

Kumagai, H. Cho, S. H. Ishikawa, K. Midorikawa, K. Fujimoto, M. Aoshima, S. Tsuchiya, Y. (2003). Observation of the comples propagation of a femtosecond laser pulse in a dispersive transparent bulk material, Journal of Optical Society of America, Vol. 20., No. 3., (March 2003).

Macke, B. Segard, B. (2003). Propagation of light pulses at a negative group velocity, European Physical Journal D, Vol. 23., (125-141).

Niikura H. (2002). Sub-laser-cycle electron pulses for probing molecular dynamics. Nature 417, (917-922).

Oughstun, K. E. Sherman, G. C. (1989). Uniform asymptotic description of electromagnetic pulse propagation in a linear dispersive medium with absorption (the Lorentz medium), Journal of Optical Society of America A, Vol. 6., No. 9., (September 1989), (1394-1420). 
Parali, Ufuk Alexander,Dennis R. (2010). Interaction of a single-cycle laser pulse with a bound electron without ionization. Optics Express. Vol. 18., No. 14., (July 2010).

Pietrzyk, M. Kanattsikov, I. Bandelow, U. (2008). On the propagation of vector ultrashort pulses, Journal of Nonlinear Mathematical Physics, Vol. 15., No. 2., (162-170).

Porras M. A. (1999). Nonsinusoidal few-cycle pulsed light beams in free space, Journal of Optical Society of America B, Vol. 16., No. 9., (September 1999).

Rothenberg, J. E. (1992). Space-time focusing: Breakdown of the slowly varying envelope approximation in the self-focusing of femtosecond pulses. Optics Letters, Vol. 17., No. 19., (October 1992)

Scaife, B.K.P. (1989). Principles of Dielectrics, Oxford University Press, Oxford.

Schaffer, C. B. (2001). Interaction of femtosecond laser pulses with transparent materials, Ph.D. Thesis. Harvard University.

Shvartsburg, A. B. (1996). Time-Domain Optics of Ultrashort Waveforms. Clarendon Press, Oxford

Shvartsburg, A. B. (1998). Single-cycle waveforms and non-periodic waves in dispersive media (exactly solvable models). Physics - Uspekhi, Vol. 41., No. 1., (77-94).

Shvartsburg, A. B. (1999). Impulse Time-Domain Electromagnetic of Continuos Media. Birkhauser Verlag, Boston.

Shvartsburg, A. B. (2005). Optics of nonstationary media, Physics - Uspekhi, Vol. 48., No. 8., (797-823)

Shvartsburg, A. B. Petite, G. (2002). Progress in Optics, Vol. 44 (Ed. E Wolf), p. 143., Elsevier Sci

Steinmeyer, G. Sutter, D. H. Gallmann, L. Matuschek, N. Keller, U. (1999). Frontiers in Ultrashort Pulse Generation: Pushing the Limits in Linear and Nonlinear Optics. Science, Vol. 286., (November 1999).

Tang, T.; Xu, X. \& Cheng, J. (2008). On spectral methods for Volterra integral equations and the convergence analysis, Journal of Computational Mathematics, Vol.26, No. 6., (825-837).

Wang, Z. Zhang, Z. Xu, Z. Lin, Q. (1997). Space-time profiles of an ultrashort pulsed Gaussian beam, IEEE Journal of Quantum Electronics, Vol. 33., No. 4., (April 1997).

Wilkelmsson, H. Trombert, J. H. Eloy, J. F. (1995). Dispersive and dissipative medium response to an ultrashort pulse: A green's function approach. Physica Scripta, Vol. 52., (102-107).

Xiao, H. Oughstun, K. E. (1999). Failure of the group velocity description for ultrawideband pulse propagation in a casually dispersive, absorptive dielectric, Journal of Optical Society of America B, Vol. 16., No. 10., (October 1999).

Yan, Y. Gamble, E. B. Jr. Nelson, K. A. (1985). Impulsive stimulated scattering: General importance in femtosecond laser pulse interactions with matter, and spectroscopic applications. J. Chem. Phys. Vol. 83., No. 11., (December 1985).

Zewail A. (2000). Femtochemistry: atomic-scale dynamics of the chemical bond, Journal of Physical Chemistry A, Vol. 104, (5660-5694).

Zou, Q. Lu, B. (2007). Propagation properties of ultrashort pulsed beams with constant waist width in free space. Optics and Laser Technology, Vol. 39, (619-625). 


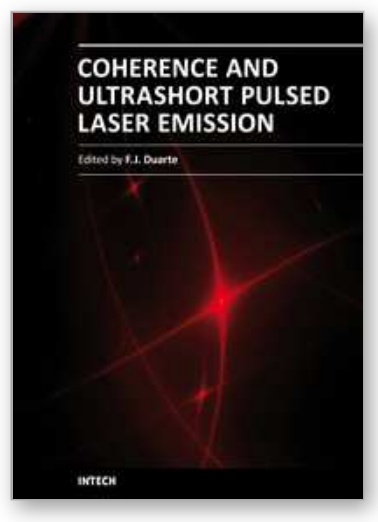

\section{Coherence and Ultrashort Pulse Laser Emission}

Edited by Dr. F. J. Duarte

ISBN 978-953-307-242-5

Hard cover, 688 pages

Publisher InTech

Published online 30, November, 2010

Published in print edition November, 2010

In this volume, recent contributions on coherence provide a useful perspective on the diversity of various coherent sources of emission and coherent related phenomena of current interest. These papers provide a preamble for a larger collection of contributions on ultrashort pulse laser generation and ultrashort pulse laser phenomena. Papers on ultrashort pulse phenomena include works on few cycle pulses, high-power generation, propagation in various media, to various applications of current interest. Undoubtedly, Coherence and Ultrashort Pulse Emission offers a rich and practical perspective on this rapidly evolving field.

\section{How to reference}

In order to correctly reference this scholarly work, feel free to copy and paste the following:

Ufuk Parali and Dennis Alexander (2010). Modeling the Interaction of a Single-Cycle Laser Pulse with a Bound Electron without Ionization, Coherence and Ultrashort Pulse Laser Emission, Dr. F. J. Duarte (Ed.), ISBN: 978953-307-242-5, InTech, Available from: http://www.intechopen.com/books/coherence-and-ultrashort-pulselaser-emission/modeling-the-interaction-of-a-single-cycle-laser-pulse-with-a-bound-electron-without-ionization

\section{INTECH}

open science | open minds

\section{InTech Europe}

University Campus STeP Ri

Slavka Krautzeka 83/A

51000 Rijeka, Croatia

Phone: +385 (51) 770447

Fax: +385 (51) 686166

www.intechopen.com

\section{InTech China}

Unit 405, Office Block, Hotel Equatorial Shanghai

No.65, Yan An Road (West), Shanghai, 200040, China

中国上海市延安西路65号上海国际贵都大饭店办公楼 405 单元

Phone: +86-21-62489820

Fax: +86-21-62489821 
(C) 2010 The Author(s). Licensee IntechOpen. This chapter is distributed under the terms of the Creative Commons Attribution-NonCommercialShareAlike-3.0 License, which permits use, distribution and reproduction for non-commercial purposes, provided the original is properly cited and derivative works building on this content are distributed under the same license. 